\title{
Pre-operative stereotactic radiosurgery treatment is preferred to post-operative treatment for smaller solitary brain metastases
}

Hamidreza Aliabadi ${ }^{*}$, Arian M. Nikpour ${ }^{2}$, David S. Yoo ${ }^{3}$, James E. Herndon $I^{4}$, John H. Sampson ${ }^{5}$ and John P. Kirkpatrick ${ }^{3}$

\begin{abstract}
Background: While the optimal combination of whole-brain radiotherapy (WBRT), stereotactic radiosurgery (SRS) and surgical resection in the treatment of brain metastases, is controversial, the addition of SRS to surgical resction of solitary metastasis may enhance local control while potentially minimizing toxicity associated with adjuvant WBRT. This study seeks to determine whether pre-operative SRS to the lesion versus post-operative SRS to the resection bed may reduce irradiation of adjacent normal brain tissue.

Methods: A retrospective study of 12 patients with 13 surgically resected cerebral metastases was performed. The pre-operative contrast-enhancing tumors and post-operative resection cavities plus any enhancing residual disease were contoured to yield the gross target volume (GTV). In turn these GTV's were uniformly expanded by 3-mm to generate the pre-operative, as well as post-operative planning target volume (PTV.) For each lesion, a 7-staticconformal-beam, non-coplanar plan utilizing $6 \mathrm{MV}$ photons was generated to encompass the PTV within the 85\% isodose line. Excess normal brain volume irradiated was defined as the volume outside the GTV receiving the prescribed dose.

Results: When lesions were divided into two groups - Group A (pre-operative GTV's $<15 \mathrm{cc}, n=9$ ) and Group B (pre-operative GTV's $>15 \mathrm{cc}, n=4$ ) - the average volume of normal brain irradiated was significantly smaller if preoperative SRS was used for treatment of lesions in Group A (9.5 vs. $16.8 \mathrm{cc}$, paired t-test, $p=0.0045$ ). In contrast, this volume was smaller for Group B lesions if post-operative SRS was used for treatment of these lesions (27.6 vs. 51.2 $\subset c, p=0.252$ ). A comparison of groups with respect to mean volume differences between pre- and post-operative SRS was significantly different (two-sample t-test $p=0.016$ ). GTV and the difference between pre- and postoperative volume were highly correlated (Pearson correlation $=-0.875, p<0.0001$ ).
\end{abstract}

Conclusions: Pre-operative treatment of smaller metastases may result in reduced radiation dose to normal tissue and, thus, reduced treatment-related morbidity compared to post-operative irradiation of the resection cavity.

Keywords: Stereotactic radiosurgery, Brain metastases, Pre-operative, Solitary brain metastases

\footnotetext{
* Correspondence: hamidneurosurg@yahoo.com

${ }^{1}$ Spine and Neurosurgery Associates, 1301 Secret Ravine Parkway, Suite 200,

Roseville, CA 95661, USA

Full list of author information is available at the end of the article
} 


\section{Background}

In 1990, Patchell et al. demonstrated that treatment outcomes of cerebral metastases were better when surgical resection was combined with WBRT [1]. Recurrence at the original site was reduced in these patients when compared with the group receiving only WBRT. Furthermore, the patients who underwent resection plus radiation survived longer with a better quality of life. However, postoperative SRS is used at many institutions in lieu of WBRT for treatment of cerebral metastases. This focal radiation technique offers potential advantages over WBRT, which may be associated with acute and delayed adverse effects from irradiation of normal brain parenchyma and which has not been shown to increase overall survival.

Admittedly, the rationale for WBRT versus radiosurgery is controversial [2]. However, WBRT has been associated with an acute detriment in quality-of-life measures [3], potential delayed neurocognitive deficits[4, 5], and lack of overall survival benefit [6-10]. However, the treatment volume of the resection cavity post-operatively when SRS treatments are used may be an overestimation of the true lesion volume requiring treatment. This conjecture is based on the often irregular shape observed for the post-operative resection cavity. In turn, this tortuosity and high aspect ratio will result in poorer conformality of the treatment plan compared and, possibly, irradiation of a greater volume of normal adjacent parenchyma than in the case of treatment of the typically "rounder and smoother" pre-operative target. Thus, there may be a significant advantage to preoperative SRS treatment instead of post-operative treatment. In addition, major benefits of pre-operative SRS may be the reduction of tumor "spill" and subsequent dissemination, as well as irradiation of a better oxygenated and, thus, more radiosensitive target.

The purpose of this study is to determine if pre-operative SRS results in less irradiation of adjacent normal brain tissue and other critical structures than post-operative SRS. Furthermore, if pre-operative SRS is advantageous to postoperative SRS, we aimed to determine which lesions should be treated with pre-operative SRS versus post-operative SRS. In addition though, pre-operative SRS, if deemed beneficial, may minimize the delay between surgery and the start of adjuvant therapy. Moreover, retrospective $[8,11$, 12 ] and prospective trials report no apparent survival benefit in patients with brain metastases when WBRT was added to SRS as opposed to SRS alone.

To the best of our knowledge, this retrospective analysis is the first published report looking at matched-pair dose distribution between pre-operative versus postoperative SRS treatments.

\section{Methods}

A retrospective review of patients with surgically resected single cerebral metastases was performed.
Selection criteria included patients with diagnoses of primary malignancy and finding of solitary brain metastasis, with exception of one case, where two lesions were contiguous and able to be removed in one sitting in one single approach. Criteria were not limited to any particular type of malignancy. Both male and female adult patients with diagnosis of primary malignancy were included as long as they had a resectable solitary metastasis, were medically cleared preoperatively, and were deemed to be surgical candidates.

Lesions located in anatomic regions that are not amenable to SRS, including the brain stem, optic apparatus, were excluded from this study. Small tumors were arbitrarily characterized as 15 cc or less, which is a volume generally small enough not to cause mass effect. All patients had a gross total resection as evidenced by a post-operative MRI scan of the brain with and without contrast followed by subsequent radiosurgery to the resection cavity. Utilizing the pre-operative MR images, the dose distribution was also calculated, as if these same patients had been treated with pre-operative SRS to the unresected lesion. Thus, the volume of normal brain parenchyma was directly compared for each patient based on hypothetical radiosurgery of the preoperative lesion versus post-operative resection bed.

All patients underwent standard-of-care pre-operative MRI of the brain with gadolinium-diethylenetriaminepentaacetate using T1-weighted 3-mm ( $\mathrm{mm}$ ) thick contiguous axial-cuts as well as MRIs within $48 \mathrm{~h}$ of surgery. Images were imported into an Eclipse treatment planning system (Varian Medical Systems, Palo Alto, CA.) Enhancing pre-resection tumors and post-operative resection cavities plus any enhancing residual disease were contoured on a slice-by-slice basis to yield the gross target volumes (GTV, Fig. 1). These GTV's were uniformly expanded by $3-\mathrm{mm}$ to generate the pre-operative and post-operative PTV's. For each lesion, a 7-static-beam, non-coplanar plan utilizing six MV photons was created to encompass the PTV within the $85 \%$ isodose line. Excess brain volume irradiated was calculated by measuring the volume of brain encompassed by the $85 \%$ isodose line and subtracting the GTV.

Lesions were classified according to their pre-operative GTV: Group A (pre-operative GTV's $<15 \mathrm{cc}, n=9$ ) and Group B (pre-operative GTVs $\geq 15$ cc, $n=4$ ). Within each group, a paired t-test compared the average volume of normal brain that would be irradiated if the treatment plan included pre-operative versus post-operative SRS. A two-sample t-test compared groups with respect to mean volume differences between pre- and postoperative SRS. Pearson and Spearman correlation coefficients were generated for the relationship between GTV and the volume of normal brain irradiated with pre- versus post-operative SRS. 


\section{Example (2), pre-operative \\ contouring}

Example (1) - pre-operative contouring
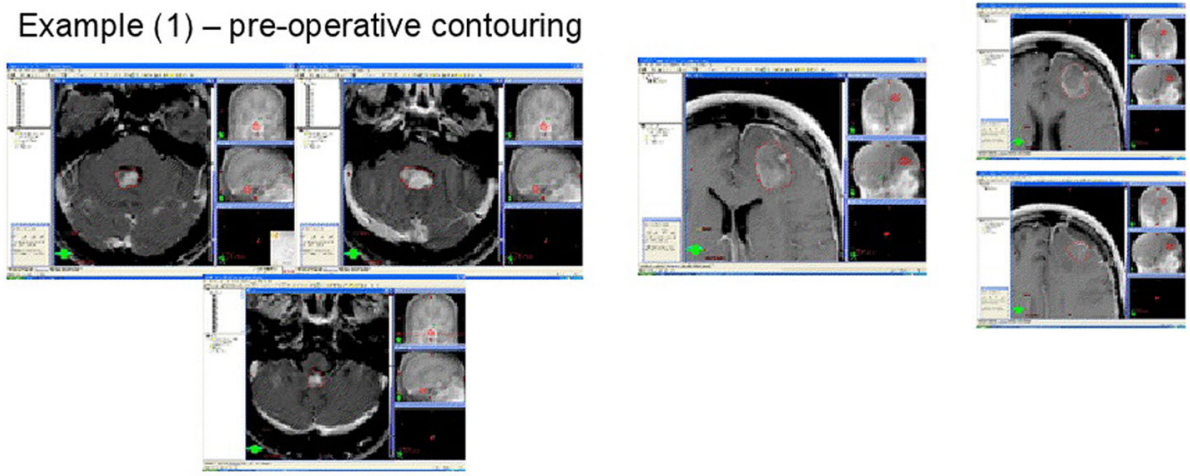

Example (2b), post-operative contouring

Example (1b)-post-operative contouring
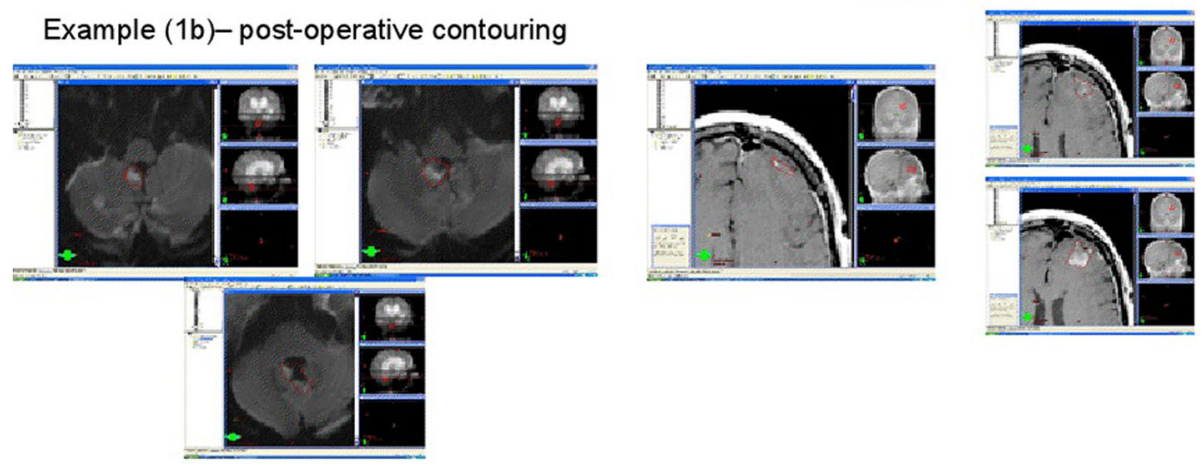

Fig. 1 Pre-operative and post-operative contouring examples

\section{Results}

We retrospectively analyzed 12 patients who presented with 13 solitary brain metastases which were resected successfully with gross total resection. In Table 1, patient characteristics (i.e., longest tumor axis $(\mathrm{cm})$, anatomic location, histopathology, and GTV of the tumors) are presented. Table 2 shows radiosurgical planning data for extrapolation of excess brain tissue irradiated during SRS treatments for both pre- and post-surgery. Finally, Table 3 shows the patients divided into two groups

Table 1 Patient characteristics

\begin{tabular}{|c|c|c|c|c|}
\hline PT\# & Pathology & Location & Tumor dimension (longest axis, cm) & GTV (cc) \\
\hline 1 & Non-small cell lung cancer (NSCLC) & right temporal & 3.9 & 14.85 \\
\hline 2 & Bladder/transitional cell & fourth ventricular & 1.8 & 3.36 \\
\hline 3 & NSCLC & left cerebellar & 4.3 & 23.89 \\
\hline 4 & Lung-papillary adenocarcinoma & left occipital & 0.8 & 0.34 \\
\hline 5 & Carcinoid & left frontal & 5.5 & 67.88 \\
\hline 6 & NSCLC & right frontal & 4.7 & 37.32 \\
\hline 7 & melanoma & left frontal & 0.3 & 0.06 \\
\hline 8 & Lung-papillary adenocarcinoma & left cerebellar & 1.9 & 2.8 \\
\hline 9 & NSCLC & right parietal & 1.7 & 1.8 \\
\hline 10 & Lung adenocarcinoma & right cerebellar & 3.7 & 0.33 \\
\hline 10 & Lung adenocarcinoma & left cerebellar & 0.7 & 17.39 \\
\hline 11 & Lung squamous cell & right frontal & 3.1 & 11.38 \\
\hline 12 & Lung adenocarcinoma & left frontal & 2.9 & 6.09 \\
\hline
\end{tabular}


based on tumor volume. Group A patients had a metastasis that was $<15 \mathrm{cc}$ in volume and these tumors were considered "small," while Group B patients had a tumor $>15 \mathrm{cc}$ in volume and these tumors were considered "large". The dosimetry was then calculated based on pre- and post-operative radiosurgery plans with associated volume of normal adjacent brain irradiated for each (i.e., pre- versus post-operative SRS).

Lesions with average pre-operative GTV of $14.0 \mathrm{cc}$ (range 0.06-67.9 cc), average pre-operative GTV plus 3$\mathrm{mm}$ margin volume of $27.1 \mathrm{cc}$ (range $0.79-117 \mathrm{cc}$ ), and pre-operative volumes encompassed by $85 \%$ isodose line of 39.9 cc (range 1.09-166 cc) were evaluated. For the overall group, there was no significant difference observed in calculated mean volume of normal brain irradiated in the pre-operative and post-operative treatment plans - 22.4 cc (range 0.97-93.5 cc) versus 20.1 cc (range 7.07-62.11 cc), respectively.

However, when lesions were divided into the two above mentioned groups: Group A (pre-operative GTV's < 15 cc, $n=9$ ) and Group B (pre-operative GTVs $\geq 15 \mathrm{cc}, n=4$ ), we found that the average volume of normal brain irradiated was significantly smaller if preoperative SRS was used for treatment of lesions in Group A (9.5 vs. 16.8 cc, paired t-test, $p=0.0045$ ) (Fig. 2). In contrast, this volume was smaller for Group B lesions if post-operative SRS was used for treatment of these lesions (27.6 vs. $51.2 \mathrm{cc}, p=0.252$ ).

The mean differences between the pre- and post-operative volume difference were computed within Groups A and B.
Table 3 Solitary metastases based on GTV (small <15 cc and large $>15 \mathrm{cc}$ ) and volume of normal adjacent brain irradiated if SRS was administered pre- versus post-operatively

\begin{tabular}{lll}
\hline & \multicolumn{2}{l}{ Normal Brain Irradiated (cc) } \\
\cline { 2 - 3 } Pre-Op SRS & Post-Op SRS \\
\hline 23.89 & & \\
67.88 & 26.16 & 13.54 \\
37.32 & 93.5 & 20.52 \\
17.39 & 61.77 & 62.11 \\
GTV $<15 c c$ & 23.49 & 14.36 \\
14.85 & & \\
3.36 & 24.51 & 29.08 \\
0.34 & 11.99 & 14.39 \\
0.06 & 1.97 & 7.34 \\
2.8 & 0.97 & 18.22 \\
1.8 & 6.73 & 13.22 \\
0.33 & 6.78 & 12.39 \\
11.38 & 2.44 & 7.07 \\
6.09 & 17.41 & 33.59 \\
\hline
\end{tabular}

A two-sample t-test showed a statistically significant group effect $(p=0.016)$. The correlation between GTV and volume of normal brain irradiated with pre-operative versus postoperative SRS was further assessed.

The parametric and non-parametric correlation estimates were statistically significant $[$ Pearson correlation $=-0.875$

Table 2 Radiosurgical planning data for extrapolation of excess brain irradiated during SRS treatments for both pre- versus postsurgery. (GTV = gross tumor volume)

\begin{tabular}{|c|c|c|c|c|c|c|c|c|c|c|c|c|}
\hline \multirow[t]{2}{*}{ PT\# } & \multirow{2}{*}{\multicolumn{2}{|c|}{ Pathology }} & \multicolumn{2}{|l|}{ GTV } & \multicolumn{2}{|c|}{$\mathrm{GTV}+3 \mathrm{~mm}$} & \multicolumn{2}{|c|}{ Isodose volume } & \multicolumn{2}{|c|}{ Excess Brain } & \multicolumn{2}{|l|}{ IDL/GTV } \\
\hline & & & PRE & POST & PRE & POST & PRE & POST & PRE & POST & PRE & POST \\
\hline 1 & NSCLC & & 14.85 & 27.67 & 35.1 & 48.79 & 52.28 & 72.19 & 24.51 & 29.08 & 3.520539 & 2.608963 \\
\hline 2 & Bladder/transitional cell & & 3.36 & 3.39 & 11.45 & 11.83 & 15.86 & 18.38 & 11.99 & 14.39 & 4.720238 & 5.421829 \\
\hline 3 & NSCLC & & 23.89 & 8.97 & 41 & 16.73 & 57.38 & 22.98 & 26.16 & 13.54 & 2.401842 & 2.561873 \\
\hline 4 & Lung-papillary adenocarcinoma & & 0.34 & 2.33 & 1.84 & 6.81 & 2.57 & 10.41 & 1.97 & 7.34 & 7.558824 & 4.467811 \\
\hline 5 & Carcinoid & & 67.88 & 6.06 & 116.62 & 17.79 & 165.83 & 27.53 & 93.5 & 20.52 & 2.442988 & 4.542904 \\
\hline 6 & NSCLC & & 37.32 & 27.81 & 63.61 & 53.71 & 101.23 & 90.5 & 61.77 & 62.11 & 2.712487 & 3.254225 \\
\hline 7 & melanoma & & 0.06 & 5.85 & 0.79 & 18.16 & 1.09 & 31.05 & 0.97 & 18.22 & 18.16667 & 5.307692 \\
\hline 8 & Lung-papillary adenocarcinoma & & 2.8 & 9.57 & 7.4 & 21.87 & 10.93 & 27.53 & 6.73 & 13.22 & 3.903571 & 2.876698 \\
\hline 9 & NSCLC & & 1.8 & 5.87 & 5.68 & 14.34 & 8.6 & 21.02 & 6.78 & 12.39 & 4.777778 & 3.58092 \\
\hline 10 & Lung adenocarcinoma & & 0.33 & 1.55 & 1.82 & 7.05 & 2.8 & 9.92 & 2.44 & 7.07 & 8.484848 & 6.4 \\
\hline 10 & Lung adenocarcinoma & & 17.39 & 4.8 & 32.3 & 17.04 & 47.78 & 24.22 & 23.49 & 14.36 & 2.747556 & 5.045833 \\
\hline 11 & Lung squamous cell & & 11.38 & 22.18 & 22.78 & 45.05 & 35.57 & 82.05 & 17.41 & 33.59 & 3.125659 & 3.699279 \\
\hline \multirow[t]{4}{*}{12} & Lung adenocarcinoma & & 6.09 & 9.64 & 14.11 & 20.74 & 22.73 & 32.16 & 13.02 & 15.6 & 3.732348 & 3.3361 \\
\hline & & $\min$ & 0.06 & 1.55 & 0.79 & 6.81 & 1.09 & 9.92 & 0.97 & 7.07 & & \\
\hline & & $\max$ & 67.88 & 27.81 & 116.62 & 53.71 & 165.83 & 90.5 & 93.5 & 62.11 & & \\
\hline & & average & 14.42231 & 10.43769 & 27.26923 & 23.07 & 40.35769 & 36.14923 & 22.36462 & 20.11 & & \\
\hline
\end{tabular}


Normal adjacent brain irradiated based on GTV for pre- and post-op SRS

in cubic centimeters

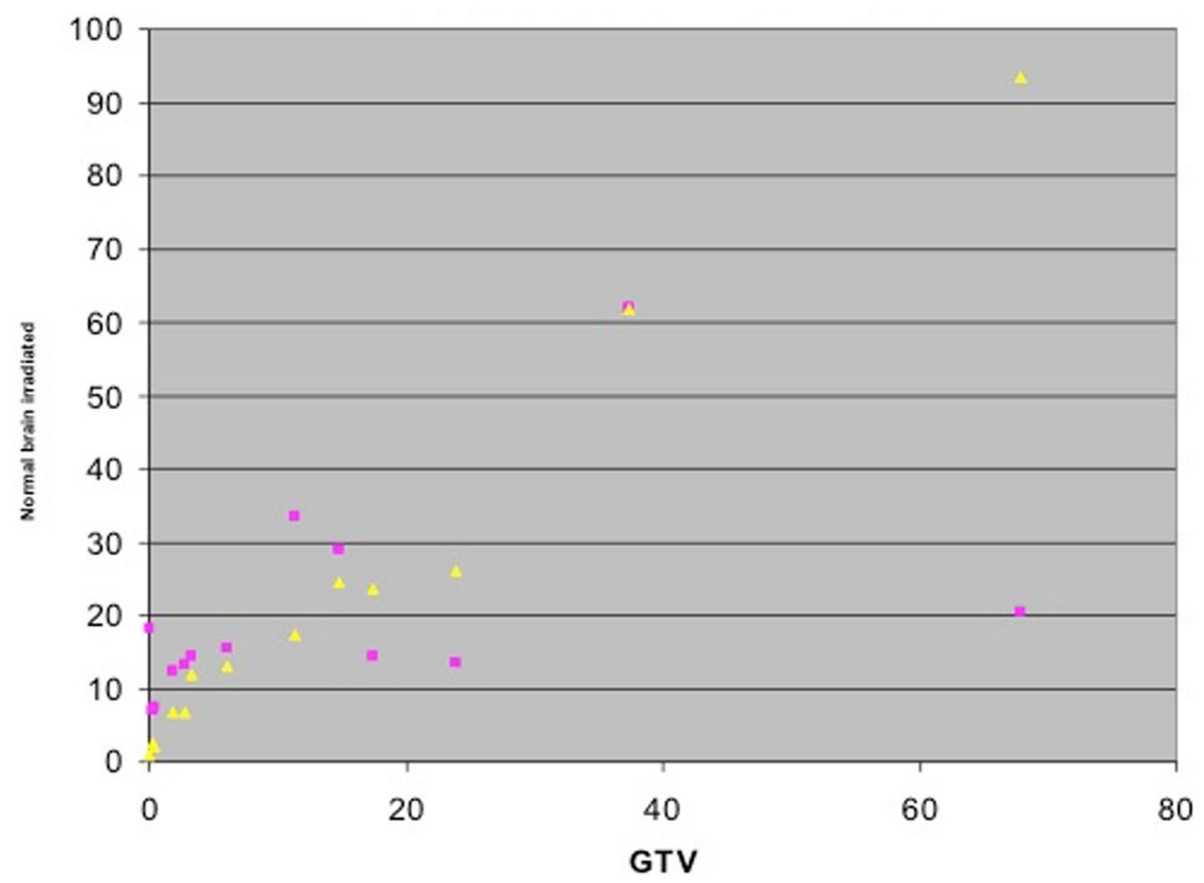

Fig. 2 Normal adjacent brain irradiated based on GTV for pre- and post-op SRS in cubic centimeters. Legend: (yellow $=$ pre-operative; pink $=$ post-operative)

$(p<0.0001)$; Spearman correlation $=-0.780(p=0.0017)]$. Therefore, pre-operative treatment of Group A tumors results in less adjacent normal brain tissue irradiation when compared to post-operative treatment. However, there is no evidence that a statistically significant difference exists for the Group B tumors.

\section{Discussion}

Brain metastases occur in $20-40 \%$ of all patients with cancer [13, 14]; 30-40\% present with a single metastasis [15]. Metastases to the brain account for the most common intracranial tumor in adults. Moreover, the incidence of brain metastases continues to rise as a result of advances in cancer diagnosis and management. It is estimated that 170,000 new cases of metastatic brain tumor are diagnosed in the United States each year. In addition, the use of MRI allows us to detect other small metastases, which would not have been visualized in the past. However, the prognosis of patients with metastases to the brain remains poor $[16,17]$.

Proper pre-treatment evaluation is important in determining the optimum treatment strategy for patients with brain metastases, which includes assessing the extent and control of systemic disease and thus identifying the appropriate staging of the disease. This evaluation is very important since patient prognosis is most based on the extent of systemic disease. At our institution, we obtain computed tomography (CT) or PET/CT scans of the chest, abdomen, and pelvis, as well as radionuclide bone scans if necessary. The extent of intracranial disease is assessed by enhanced MRI.

The presence of advanced or progressing systemic disease is a poor prognostic factor of patient survival. In such patients, whose survival may be 2 months or less, the addition of surgical resection or radiosurgery in addition to WBRT has little impact on final patient outcome. However, if the patient has stable systemic disease and brain metastases are well controlled, the survival is significantly improved. The prognosis of patients with a single brain metastasis appears to be much better than those with multiple metastases $[18,19]$. Therefore, treatment of such patients is often more aggressive and includes focal therapy, such as surgical resection or SRS in combination with WBRT. In addition, combining modalities is conceptually appealing if subpopulations of tumor cells are differentially sensitive to the different modalities.

Furthermore, SRS may avoid the acute and delayed effects of WBRT, including neurocognitive decline. In addition, SRS requires a much shorter elapsed time for treatment, and reduces the volume of normal brain parenchyma irradiated [20]. SRS is often offered to patients with a good performance status and $\leq 3$ metastases of $\leq 4 \mathrm{~cm}$ in maximum dimension [21, 22]. Historically, our practice has been to offer surgery for solitary brain 
metastases when the tumor is surgically accessible, followed by either WBRT or SRS alone, depending on the size and number of the lesions, the patient's performance status, the need for chemotherapy and the completeness of the resection When SRS is selected as the treatment modality, the neurosurgeon, radiation oncologist, and radiation physicist work together to perform target delineation, dose selection, and radiosurgical planning.

As discussed in the paper by Soltys et al. [6], SRS alone to the resection cavity could be justified as they yield a local control rate similar to that of post-operative WBRT. It has been shown by Patchell et al. in 1998 that when a gross totally resected isolated metastasis was treated with post-operative WBRT versus no additional therapy, the WBRT decreased the rate of local failure at the original tumor site from 46 to 10\% [10]. Furthermore, SRS appears to be as efficacious as WBRT to the resection cavity thus there is a role for SRS postoperatively when compared to WBRT post-operatively. In the 2008 study by Soltys et al. at Stanford University Medical Center, the local failure rate was $14 \%$ when using SRS to the resection cavity as opposed to $46 \%$ with surgery alone and $10^{10}$ to $20 \%$ [1] in patients with surgical resection followed by WBRT. In the Patchell study, the surgery alone group had a distant failure in the brain of approximately $40 \%$. It is not clear if this rate would be any different in surgically treated patients who underwent SRS treatment. However, these patients would be eligible for salvage therapies such as SRS, surgical resection and/or WBRT. Pre-operative treatment with SRS may reduce this number due to the "lack of spillage" of tumor cells.

Whole brain radiotherapy has been the "traditional" mainstay of therapy for intracranial metastases. WBRT has been shown to improve both neurological function and survival. Early non-randomized studies report that WBRT improves survival by 3-6 months [16, 23, 24]. The radiographic and clinical response rate from WBRT for intracerebral metastases varies from 64 to $85 \%$, however any clinical benefit is almost always transient. Despite WBRT, up to $50 \%$ of these patients eventually die from progression of their intracerebral disease [24, 25]. The Cochrane Review of evidence based medicine has recently reviewed nine published trials have examined various scheduled of WBRT for multiple intracranial metastases [26]. The most common instituted treatment regimen is a total of $30 \mathrm{cGy}$ delivered in ten fractions.

Comparing various outcomes including survival and neurological symptoms control, no significant differences were noted with any of the various fractionation schemes. Two randomized trials have shown that surgical resection combined with WBRT is superior to WBRT treatment alone $[1,27]$ though a third published in the Journal of the American Medical Association by
Mintz et al. failed to show an advantage. Post-operative radiotherapy is felt to kill tumor cells remaining in the operative bed as well as micrometastases in other areas of the brain. However, the routine use of post-operative WBRT may not be necessary considering a single metastasis may totally be resected, and, since these lesions do not infiltrate the adjacent parenchyma like primary CNS tumors, close follow-up and effective salvage therapy may produce equivalent overall survival rates. Thus, the appropriate administration of SRS to the lesion or resection bed may offer equivalent overall survival with reduced morbidity compared to adjuvant WBRT.

A single-fraction SRS boost is currently a viable treatment option of intracranial metastatic lesions. Several large randomized trials by the RTOG have examined the role of SRS in the management of single and multiple intracranial metastatic tumors. Additionally, SRS is well tolerated with few potential complications. Risks of SRS are minimal but primarily include complications associated radiation-related cerebral edema and radionecrosis.

In a large multi-institutional review of SRS alone vs. WBRT in the initial management of intracranial metastatic disease, Sneed et al., reported on 569 patients, 268 with SRS alone and 301 with SRS and WBRT. In this study, the use of upfront WBRT did not affect overall survival in patients treated with SRS in any of the three recursive partitioning analysis (RPA) classes (15.2 vs. 14.0 months for RPA class $1,7.0$ vs. 8.2 for class 2 , and 5.5 vs. 5.3 for class 3) [28]. In the recent study by Aoyama et al. [9], compared with SRS alone, the use of combined WBRT and SRS did not improve survival in patients with 1 to 4 brain metastases; however, recurrence occurred more commonly in patients who received SRS alone as opposed to both SRS and WBRT. Most recently, RTOG-9508 was a randomized trial which compared WBRT with or without SRS boost in patients with 1-3 brain metastases. In this trial, 331 patients were randomized to receive either WBRT alone (2.5 Gy fractions to a total of 37.5 Gy over 3 weeks) or WBRT plus a SRS boost to the tumor site (size-dependent dose, 24 Gy up to $2 \mathrm{~cm}$, $18 \mathrm{~Gy}$ for $2-3 \mathrm{~cm}$, and 15 Gy to $3-4 \mathrm{~cm}$ lesions). Ten percent of these patients had a breast primary. In this study, although the addition of SRS led to a significant improvement in local control rate, there was no survival benefit noted in patients with multiple intracranial metastases, however, in patients with a single intracranial metastasis, there was a modest, although significant survival benefit of WBRT plus SRS (4.9 months vs. 6.5 months, $p=0.03)$. Additionally, in this study, with respect to improvement in clinical function, KPS was improved at 6 months in 13\% of patients treated with 
WBRT plus SRS boost versus only $4 \%$ in patients treated with WBRT alone [29].

In patients with solitary brain metastases, WBRT potentially produces significant side-effects, especially in terms of neurocognition, without apparent improvement in overall survival. Conversely, WBRT reduces the rate of appearance of distant brain metastases which may reduce neurocognitive benefits [2]. However, given early detection of brain metastases, effective intracranial salvage therapy and improved systemic control of malignant disease, one could argue that it would be advantageous to locally treat disease with SRS and surgery alone. Patients who had solitary brain metastases underwent gross total resection for histological confirmation or mass effect without WBRT treatment but instead with post-operative SRS. However, as explained above, the treatment volume of the resection cavity post-operatively may be an overestimation of the true lesion volume requiring treatment. Since the resection cavity volume results in irradiation of a greater volume of normal adjacent parenchyma than is necessary. Thus, there may be a significant advantage to pre-operative SRS instead. Of note, the timing of the post-operative MRI may be significant. In our study, a post-operative MRI was performed immediately after surgery. The resection cavity may shrink over several weeks and thus, the timing of radiosurgery may alter study conclusions.

A lesion's size, but not shape, may allows us to predict whether pre-operative versus post-operative SRS treatment is beneficial to a patient with solitary brain metastases. The ability to predict the GTV of a lesion preoperatively versus post-operatively allows us to determine when SRS treatments should be administered (i.e., before surgical resection or afterwards). Unfortunately, there is no easy way to make such a prediction; however, it appears that the only reliable way to determine whether or not a lesion's pre-operative GTV would be less than its post-operative GTV would be if its preoperative GTV is $<15 \mathrm{cc}$. We found that when the preoperative GTV was smaller than the post-operative GTV (i.e., pre-operative GTV < 15 cc), pre-operative SRS may result in reduced radiation dose to normal tissue, potentially reducing treatment-related morbidity compared to post-operative irradiation of the resection cavity. However, further data are needed to determine if postoperative irradiation may be dosimetrically favored for larger lesions.

\section{Conclusions}

Pre-operative treatment of smaller metastases may result in reduced radiation dose to normal tissue and, thus, reduced treatment-related morbidity compared to postoperative irradiation of the resection cavity of intracranial metastasis.

\section{Abbreviations}

GTV: Gross target volume; KPS: Karnofsky Performance Scale; MRI: Magnetic resonance imaging; PTV: Planning target volume; RTOG: Radiation Therapy Oncology Group; SRS: Stereotactic radiosurgery; WBRT: Whole-brain radiotherapy

\section{Acknowledgments \\ Not applicable.}

\section{Funding}

No funding was provided to the authors or the institution for this study.

\section{Availability of data and materials}

Data obtained from Duke University Hospital. The data that support the findings of this study are available on request from the corresponding author. The data are not publically available due to patient confidentiality restrictions.

\section{Authors' contributions}

Preparation of manuscript, data collection, and final edits: HA, AMN. Data and statistical analysis: JEH. Study design: HA, AMN, DSY, JHS, JPK. All authors read and approved the final manuscript.

\section{Ethics approval and consent to participate}

Reviewed and approved by ethics committee and Institutional Review Board (IRB) at Duke University Hospital. This study is an ethics/RB-approved study with Approval/Reference Number (Protocol ID): Pro00006870.

\section{Consent for publication}

Reviewed and approved by ethics committee and Institutional Review Board at Duke University Hospital for publication of patient clinical data. We have also obtained written consent from all of the patients involved in this study.

\section{Competing interests}

The authors declare that they have no competing interests.

\section{Author details}

${ }^{1}$ Spine and Neurosurgery Associates, 1301 Secret Ravine Parkway, Suite 200, Roseville, CA 95661, USA. 'Department of Radiology, UC Davis Medical Center, UC Davis School of Medicine, Sacramento, CA, USA. ${ }^{3}$ Department of Radiation Oncology, Duke University Medical Center, Durham, NC, USA.

${ }^{4}$ Department of Biostatistics and Bioinformatics, Duke University Medical Center, Durham, NC, USA. ${ }^{5}$ Department of Surgery, Division of Neurosurgery, Duke University Medical Center, Durham, NC, USA.

Received: 18 August 2016 Accepted: 27 July 2017

Published online: 03 October 2017

\section{References}

1. Andrews DW, Scott CB, Sperduto PW, Flanders AE, Gaspar LE, et al. Whole brain radiation therapy with or without stereotactic radiosurgery boost for patients with one to three brain metastases: phase III results of the RTOG 9508 randomised trial. Lancet. 2004:363:1665-72.

2. Aoyama H, Shirato H, Tago M, Nakagawa K, Toyoda T, et al. Stereotactic radiosurgery plus whole-brain radiation therapy vs stereotactic radiosurgery alone for treatment of brain metastases: a randomized controlled trial. JAMA. 2006:295:2483-91.

3. Auchter RM, Lamond JP, Alexander E, Buatti JM, Chappell R, et al. A multiinstitutional outcome and prognostic factor analysis of radiosurgery for resectable single brain metastasis. Int J Radiat Oncol Biol Phys. 1996;35:27-35.

4. Bradley KA, Mehta MP. Management of brain metastases. Semin Oncol. 2004;31:693-701.

5. Borgelt B, Gelber R, Kramer $S$, et al. The palliation of brain metastases: final results of the first two studies by the radiation therapy oncology group. Int J Radiat Oncol Biol Phys. 1980;6:1-9.

6. Chidel MA, Suh JH, Reddy CA, Chao ST, Lundbeck MF, et al. Application of recursive partitioning analysis and evaluation of the use of whole brain radiation among patients treated with stereotactic radiosurgery for newly diagnosed brain metastases. Int J Radiat Oncol Biol Phys. 2000;47:993-9. 
7. Chow E, Davis L, Holden L, Tsao M, Danjoux C. Prospective assessment of patient-rated symptoms following whole brain radiotherapy for brain metastases. J Pain Symptom Manag. 2005;30:18-23.

8. DeAngelis LM, Delattre JY, Posner JB. Radiation-induced dementia in patients cured of brain metastases. Neurology. 1989;39:789-96.

9. Katz HR. The relative effectiveness of radiation therapy, corticosteroids, and surgery in the management of melanoma metastatic to the central nervous system. Int J Radiat Oncol Biol Phys. 1981;7:897-906.

10. Kondziolka D, Niranjan A, Flickinger JC, Lunsford LD. Radiosurgery with or without whole-brain radiotherapy for brain metastases: the patients' perspective regarding complications. Am J Clin Oncol. 2005;28:173-9.

11. Lohr F, Pirzkall A, Hof H, Fleckenstein K, Debus J. Adjuvant treatment of brain metastases. Semin Surg Oncol. 2001;20:50-6.

12. Nieder C, Nestle U, Motaref B, Walter K, Niewald M, et al. Prognostic factors in brain metastases: should patients be selected for aggressive treatment according to recursive partitioning analysis (RPA) classes? Int J Radiat Oncol Biol Phys. 2000;46:297-302.

13. Nieder C, Schwerdtfeger K, Steudel WI, Schnabel K. Patterns of relapse and late toxicity after resection and whole-brain radiotherapy for solitary brain metastases. Strahlenther Onkol. 1998;174:275-8.

14. Patchell RA. The management of brain metastases. Cancer Treat Rev. 2003; 29:533-40.

15. Patchell RA, Tibbs PA, Regine WF, Dempsey RJ, Mohiuddin M, et al. Postoperative radiotherapy in the treatment of single metastases to the brain: a randomized trial. JAMA. 1998;280:1485-9.

16. Patchell RA, Tibbs PA, Walsh JW, Dempsey RJ, Maruyama Y, et al. A randomized trial of surgery in the treatment of single metastases to the brain. N Engl J Med. 1990;322:494-500.

17. Posner JB. Management of central nervous system metastases. Semin Oncol. 1977:4:81-91.

18. Rades D, Bohlen G, Pluemer A, Veninga T, Hanssens P, et al. Stereotactic radiosurgery alone versus resection plus whole-brain radiotherapy for 1 or 2 brain metastases in recursive partitioning analysis class 1 and 2 patients. Cancer. 2007;109:2515-21.

19. Shiau CY, Sneed PK, Shu HK, Lamborn KR, McDermott MW, et al. Radiosurgery for brain metastases: relationship of dose and pattern of enhancement to local control. Int J Radiat Oncol Biol Phys. 1997;37:375-83.

20. Sneed PK, Lamborn KR, Forstner JM, McDermott MW, Chang S, et al. Radiosurgery for brain metastases: is whole brain radiotherapy necessary? Int J Radiat Oncol Biol Phys. 1999;43:549-58.

21. Sneed PK, Larson DA, Wara WM. Radiotherapy for cerebral metastases. Neurosurg Clin N Am. 1996;7:505-15.

22. Sneed PK, Suh JH, Goetsch SJ, Sanghavi SN, Chappell R, et al. A multiinstitutional review of radiosurgery alone vs. radiosurgery with whole brain radiotherapy as the initial management of brain metastases. Int J Radiat Oncol Biol Phys. 2002;53:519-26.

23. Soltys SG, Adler JR, Lipani JD, Jackson PS, Choi CY, et al. Stereotactic radiosurgery of the postoperative resection cavity for brain metastases. Int J Radiat Oncol Biol Phys. 2008;70:187-93.

24. Suh JH. Stereotactic radiosurgery for the management of brain metastases. N Engl J Med. 2010;362:1119-27.

25. Sundstrom JT, Minn H, Lertola KK, Nordman E. Prognosis of patients treated for intracranial metastases with whole-brain irradiation. Ann Med. 1998;30:296-9.

26. Tsao MN, Lloyd N, Wong R, Chow E, Rakovitch E, et al. Whole brain radiotherapy for the treatment of multiple brain metastases. Cochrane Database Syst Rev. 2006;3:CD003869.

27. Vecht CJ, Haaxma-Reiche H, Noordijk EM, Padberg GW, Voormolen JH, et al. Treatment of single brain metastasis: radiotherapy alone or combined with neurosurgery? Ann Neurol. 1993;33:583-90.

28. Weltman E, Salvajoli JV, Brandt RA, de Morais HR, Prisco FE, et al. Radiosurgery for brain metastases: a score index for predicting prognosis. Int J Radiat Oncol Biol Phys. 2000;46:1155-61.

29. Zimm S, Wampler GL, Stablein D, Hazra T, Young HF. Intracerebral metastases in solid-tumor patients: natural history and results of treatment. Cancer. 1981;48:384-94.

\section{Submit your next manuscript to BioMed Central and we will help you at every step:}

- We accept pre-submission inquiries

- Our selector tool helps you to find the most relevant journal

- We provide round the clock customer support

- Convenient online submission

- Thorough peer review

- Inclusion in PubMed and all major indexing services

- Maximum visibility for your research

Submit your manuscript at www.biomedcentral.com/submit
Biomed Central 